\title{
Atom-atom interactions at and between metal surfaces at nonzero temperature
}

\author{
M. Boström, J. J. Longdell, and B. W. Ninham* \\ Department of Applied Mathematics, Research School of Physical Sciences and Engineering, Institute of Advanced Studies, \\ Australian National University, Canberra, Australia 0200 \\ (Received 17 May 2001; published 13 November 2001)
}

\begin{abstract}
We have investigated the temperature-dependent Casimir-Polder interaction between two oscillators in the proximity of metal surfaces. The interaction near a single metal surface has much in common with the interaction in free space. However, at any finite temperature the long-range asymptote is equal to the hightemperature asymptote. This asymptote, which originates not from the $n=0$ term in the Matsubara summation but from thermal population of the $n>0$ terms, is $F(R)=-2 k_{B} T \alpha_{0}^{2} / R^{6}$. This should be compared with the more rapidly decaying zero-temperature Casimir-Polder asymptote, $F(R) \approx-13 \hbar c \alpha_{0}^{2} /\left(2 \pi R^{7}\right)$. The interaction in the midplane between two metallic surfaces is very different. The nonretarded interaction decreases exponentially and the interaction is dominated by an enhanced Casimir-Polder-like asymptote. At large separations this asymptote also decays exponentially. For any relevant temperatures the long-range asymptote is no longer equal to the high-temperature limit. In other words crossover to a classical limit found for the longrange interaction in free space, and on a metal surface, is not always valid in a narrow cavity.
\end{abstract}

DOI: 10.1103/PhysRevA.64.062702

PACS number(s): 34.20.Cf, 03.70.+k, 11.10.Wx, 34.50.Dy

\section{INTRODUCTION}

There has recently been an increased interest in fluctuation-induced forces. The forces predicted by Casimir [1] between metal surfaces and by Casimir and Polder [2] between atoms have come to play a vital role in the understanding of quantum electrodynamics. Such forces may well have fundamental importance in chemical physics and in biochemistry. Dispersion forces acting on ions have been invoked to explain the ion specific surface tension of salt solutions $[3,4]$. The classical theory of colloidal particle interactions is fundamentally in error due to the omission of such forces in the theory $[3,5]$. Our interest is in the effect of temperature on atomic interactions [6,7] near and between metallic boundaries. At any nonzero temperature, the Casimir and Casimir-Polder interactions take on a different form to that at zero temperature, hence it is of interest from a fundamental point of view. It is also of interest for applications such as catalysis. Lundström et al. have for instance investigated the influence of boundaries on catalysis in channels with depths down to $100 \mathrm{~nm}[8,9]$. $\mathrm{NO}_{x}$ are very undesirable byproducts of high-temperature combustion, and catalytic experiments aiming to solve this problem have involved nanometer sized slits and pores [10]. There are many different interactions involved in understanding catalysis, but one important contribution certainly comes from the dispersion forces [11] of the kind investigated in this work.

Mahanty and Ninham $[12,13]$ showed that there are important boundary effects on the dispersion interaction between a pair of oscillators. Vigoureux et al. [14] investigated the polarizability and dispersion interaction (in the nonretarded limit) between a pair of adsorbed atoms. There have also been calculations performed on cavity quantum electrodynamics between parallel dielectric surfaces [15-17]. Con-

\footnotetext{
*Present address: Malmö University, School of Technology and Society, SE-205 06 Malmö, Sweden.
}

finement influences both van der Waals interactions, the decay rates of atoms in excited states, and the resonance interaction $[18,19]$.

A review of both theoretical and experimental work done on these forces before 1993 is given in Ref. [20]. Not only have the measurements of the Casimir force between metal surfaces [21-23] and the Casimir-Polder force between atom and surfaces [24-29] verified the existence of these forces, but they have also been used to put up constraints on forces predicted by unified gauge theories [30]. At finite temperature thermal population of the electromagnetic field modes influences the interaction. In spite of all the efforts to measure these forces no one has so far managed to measure thermal corrections to the Casimir force, nor to the CasimirPolder force.

Wennerström et al. [7] argued that the usual interpretation of retardation effects in terms of losses in interatomic correlations due to the finite velocity of light is too simplistic, indeed erroreous! Instead they proposed an alternative interpretation in terms of thermal population of the electromagnetic field. At high enough temperatures the interaction between two atoms goes over to its classical analog. As pointed out in Ref. [7] it is quite remarkable that the same system at any finite temperature goes to the same limit at large enough separations. They used both the Lifshitz theory and fourthorder quantum electrodynamic perturbation theory to obtain asymptotic results for the interaction between two atoms. Similar results were obtained by Goedecke and Wood [31], and by Boyer [32].

In the present work we discuss the influence of retardation and finite temperature on the interaction free energy between two harmonic oscillators in a cavity with metallic walls. In Sec. II we present the general theory. We will then consider two limiting cases: both oscillators on a single surface in Sec. III, and both atoms in the midplane of a cavity in Sec. IV. We show that while the interaction on a surface has many things in common with interaction in free space, in a narrow cavity the situation is very different. We restrict our investi- 
gation to where the cavity is narrow compared to both the interparticle separation and $\hbar c /\left(k_{B} T\right)$. In a cavity that is that narrow, the nonretarded interaction decays exponentially and the interaction is dominated by a Casimir-Polder-like asymptote. At large enough separations this term decays exponentially also. In addition to this we find that the interaction, unlike in free space, does not go to a classical (where all the fluctuations are thermal) limit at large separations. Thus we can conclude that it is important in both cases to consider retardation and thermodynamic effects together to find the correct long-range interaction. Some aspects of the interaction between two atoms near a metal surface and in a narrow slit are highlighted using numerical computations in Sec. V. Finally, in Sec. VI we end with a short summary.

\section{CASIMIR-POLDER INTERACTION IN A CAVITY}

The dispersion interaction between two atoms is found as the difference between the free energy of the coupled system and that of two single atoms in a cavity. The boundary effects enter through the structure of the Green's functions of the electromagnetic field that determine the coupling between the atoms. This has been described in great detail in Ref. [13]. We will in the subsequent calculations make two simplifying assumptions. First, we will model the atoms as isotropic oscillators, second we will assume the cavity walls to be perfectly conducting although it is straightforward to extend the formalism to real metal surfaces described by local or nonlocal dielectric-response functions [13,33-35]. Work is in progress to go beyond these limitations for the interaction between ground-state atoms, and also atoms in excited configurations. We feel confident that many more interesting results will be found when this is done. Clearly, some effects such as the torque of alignment, or spin flip, due to dispersion interactions can only be investigated when anisotropy is included in the formalism. There will also be corrections to the results derived here due to for instance the finite plasma frequency of real metal surfaces.

The equations of motion for two isotropic oscillators with charge $(-e)$, mass $m$, and natural frequency $\omega_{0}$ are (after Fourier transformation) $[12,13]$ :

$$
m\left(\omega_{0}^{2}-\omega^{2}\right) \mathbf{u}_{j}(\omega)=\frac{i \omega e}{c} \mathcal{A}\left(\mathbf{R}_{j}, \omega\right)+e \boldsymbol{\nabla} \phi\left(\mathbf{R}_{j}, \omega\right) ; \quad j=1,2
$$

Maxwell's equations (in the Coulomb gauge) give,

$$
\begin{gathered}
\left(\boldsymbol{\nabla}^{2}+\frac{\omega^{2}}{c^{2}}\right) \mathcal{A}(\mathbf{r}, \omega)=\frac{i \omega}{c} \boldsymbol{\nabla} \phi+\frac{4 \pi i \omega e}{c} \sum_{j} \mathbf{u}_{j}(\omega) \delta\left(\mathbf{r}, \mathbf{R}_{j}\right) \\
\boldsymbol{\nabla} \cdot \mathcal{A}=0 \\
\boldsymbol{\nabla}^{2} \phi=4 \pi e \sum_{j} \boldsymbol{\nabla}_{R_{j}} \delta\left(\mathbf{r}, \mathbf{R}_{j}\right) \cdot \mathbf{u}_{j}(\omega)
\end{gathered}
$$

Solving Eqs. (2), (3), and (4) for the vector and scalar potentials $\mathcal{A}$ and $\phi$, and substituting the result into Eq. (1) gives the secular determinant for the coupled system. This can be rewritten as

$$
\begin{aligned}
D_{12}(\omega)= & {\left[m\left(\omega_{0}^{2}-\omega^{2}\right) \mathbf{I}+4 \pi e^{2} \mathcal{G}\left(\mathbf{R}_{1}, \mathbf{R}_{1} ; \omega\right)\right] } \\
& \times\left[m\left(\omega_{0}^{2}-\omega^{2}\right) \mathbf{I}+4 \pi e^{2} \mathcal{G}\left(\mathbf{R}_{2}, \mathbf{R}_{2} ; \omega\right)\right] \\
& -16 \pi^{2} e^{4} \mathcal{G}\left(\mathbf{R}_{1}, \mathbf{R}_{2} ; \omega\right) \mathcal{G}\left(\mathbf{R}_{2}, \mathbf{R}_{1} ; \omega\right) .
\end{aligned}
$$

The diadic Green's function $\mathcal{G}\left(\mathbf{r}, \mathbf{r}^{\prime} ; \omega\right)$ is given by,

$$
\mathcal{G}\left(\mathbf{r}, \mathbf{r}^{\prime} ; \omega\right)=\frac{\omega^{2}}{c^{2}} G^{(2)}\left(\mathbf{r}, \mathbf{r}^{\prime} ; \omega\right)-\nabla \nabla^{\prime} G^{(1)}\left(\mathbf{r}, \mathbf{r}^{\prime}\right)
$$

Here $G^{(1)}\left(\mathbf{r}, \mathbf{r}^{\prime}\right)$ is the Green's function of the equation

$$
\nabla^{2} \phi=0,
$$

and $G^{(2)}\left(\mathbf{r}, \mathbf{r}^{\prime} ; \omega\right)$ is the diadic Green's function to the equation $[12,13]$,

$$
\left(\nabla^{2}+\frac{\omega^{2}}{c^{2}}\right) \mathcal{A}=0,
$$

with the appropriate boundary conditions.

The result for two atoms shifts the frequency of the normal modes to the zeros of $D_{12}(\omega)$. Each mode contributes $\hbar \omega / 2$ to the zero-point energy and $k_{B} T \ln \left[\sinh \left(\beta \hbar \omega_{j} / 2\right)\right]$ to the free energy (here $1 / \beta=k_{B} T$ ). In order to find the total (free) energy one needs to calculate a sum over $\omega_{j}$, the zeros of $D(\omega)$. Assuming that the functions $g(z)$ and $D(z)$ are analytic this can be carried out using the identity,

$$
\sum_{\omega_{j}} g\left(\omega_{j}\right)=\frac{1}{2 \pi i} \int_{C} g(\omega) \frac{1}{D(\omega)} \frac{d D(\omega)}{d \omega} d \omega .
$$

Here $g\left(\omega_{j}\right)$ is the (free) energy of each mode. The contour $C$ includes the relevant zeros of $D(\omega)$ and excludes the poles of $g(\omega)$. Lifshitz [36] and later Ninham et al. [37] showed that such an expression for the free energy $\{g(\omega)$ $\left.=k_{B} T \ln [\sinh (\beta \hbar \omega / 2)]\right\}$ can be rewritten as a Matsubara summation over discrete frequencies

$$
F(T)=k_{B} T \sum_{n=0}^{\infty}{ }^{\prime} \ln D\left(i \xi_{n}\right), \quad \xi_{n}=2 \pi k_{B} T n / \hbar .
$$

The prime on the summation indicates that a weight of $1 / 2$ should be applied to the $n=0$ term. This term is of special importance. In many cases a term that is identical to the $n$ $=0$ term dominates the long-range interaction at finite temperatures at large enough separations. This is for instance the case for the Casimir interaction between two planar plates $[36,38,39]$, between atoms in free space $[7,31]$, and between quantum wells [40]. As we will discuss later the interaction between two atoms in a thin slit provides a challenging exception.

In our case we are interested in the changes to the free energy brought about by having both oscillators interacting 
with the field. To arrive at this relative energy we subtract the free energy arrived at from setting $D(\omega)=D_{1}(\omega) D_{2}(\omega)$ (the self-energy of two isolated oscillators) from that arrived at from setting $D(\omega)=D_{12}(\omega)$, where

$$
D_{j}(\omega)=\left|m\left(\omega_{0}^{2}-\omega^{2}\right) \mathbf{I}+4 \pi e^{2} \mathcal{G}\left(\mathbf{R}_{j}, \mathbf{R}_{j} ; \omega\right)\right| .
$$

This gives the free energy as

$$
F=k_{B} T \sum_{n=0}^{\infty} \ln \left(\frac{D_{12}\left(i \xi_{n}\right)}{D_{1}\left(i \xi_{n}\right) D_{2}\left(i \xi_{n}\right)}\right)
$$

To order $\left(e^{4}\right)$ this gives

$$
\begin{aligned}
F= & -k_{B} T \sum_{n=0}^{\infty},\left(\frac{4 \pi e^{2}}{m\left(\omega_{0}^{2}+\xi_{n}^{2}\right)}\right)^{2} \\
& \times \operatorname{Tr}\left[\mathcal{G}\left(\mathbf{R}_{1}, \mathbf{R}_{2} ;-i \xi_{n}\right) \mathcal{G}\left(\mathbf{R}_{2}, \mathbf{R}_{1} ;-i \xi_{n}\right)\right] .
\end{aligned}
$$

In this expression we immediately identify the usual approximation for the atomic polarizability of a ground-state atom, $\alpha\left(i \xi_{n}\right)=e^{2} /\left[m\left(\omega_{0}^{2}+\xi_{n}^{2}\right)\right]$.

The Green's functions for two atoms between two parallel metal plates were worked out by Mahanty and Ninham [12]. We arrive at the Green's functions for two atoms on a metal surface, and in the midplane between two metal surfaces, as limiting cases of this. They used a coordinate system where the $r_{1}$ and $r_{2}$ axis lay at one metal surface and the other surface was at $r_{3}=L$. The matrix elements for the Green's function $\mathcal{G}\left(\mathbf{r}, \mathbf{r}^{\prime} ; \omega\right)$ are $(\alpha, \beta=1,2,3)$

$$
\begin{gathered}
T_{\alpha \beta}=\left(\frac{\omega^{2}}{c^{2}} \delta_{\alpha \beta}-\frac{\partial^{2}}{\partial r_{\alpha} \partial r_{\beta}^{\prime}}\right) g_{1}\left(\mathbf{r}, \mathbf{r}^{\prime} ; \omega\right)+\frac{\omega^{2}}{c^{2}} \delta_{\alpha 3} \delta_{\beta 3} g_{2}\left(\mathbf{r}, \mathbf{r}^{\prime} ; \omega\right) ; \\
g_{1}\left(\mathbf{r}, \mathbf{r}^{\prime} ; \omega\right)=\frac{1}{2 \pi^{2} L} \sum_{p=1}^{\infty} \sin \left(\frac{p \pi r_{3}}{L}\right) \sin \left(\frac{p \pi r_{3}^{\prime}}{L}\right) \int_{-\infty}^{\infty} \int_{-\infty}^{\infty} \frac{d k_{1} d k_{2} \exp \left\{i\left[k_{1}\left(r_{1}-r_{1}^{\prime}\right)+k_{2}\left(r_{2}-r_{2}^{\prime}\right)\right]\right\}}{\omega^{2} / c^{2}-k_{p}^{2}} \\
g_{2}\left(\mathbf{r}, \mathbf{r}^{\prime} ; \omega\right)=\frac{1}{2 \pi^{2} L} \sum_{p=0}^{\infty} \cos \left(\frac{p \pi\left(r_{3}+r_{3}^{\prime}\right)}{L}\right) \int_{-\infty}^{\infty} \int_{-\infty}^{\infty} \frac{d k_{1} d k_{2} \exp \left\{i\left[k_{1}\left(r_{1}-r_{1}^{\prime}\right)+k_{2}\left(r_{2}-r_{2}^{\prime}\right)\right]\right\}}{\omega^{2} / c^{2}-k_{p}^{2}}
\end{gathered}
$$

and $k_{p}^{2}=k_{1}^{2}+k_{2}^{2}+p^{2} \pi^{2} / L^{2}$.

\section{ATOMS AT A METAL SURFACE}

We will now apply the formalism developed above to the special case of two oscillators at a metal surface. Mahanty and Ninham [12] pointed out that the nonretarded (retarded) interaction becomes reduced (enhanced) in a narrow channel compared to the free space value. We find similar results when the atoms are on a metal surface. The total retarded free energy of interaction for this case is

$$
F(\rho, T)=-16 \pi^{2} \alpha_{0}^{2} k_{B} T \sum_{n=0}^{\infty} \frac{T_{33}^{2}}{\left(1+A n^{2}\right)^{2}},
$$

where $A=\left(\xi_{1} / \omega_{0}\right)^{2}, \alpha_{0}=e^{2} / m \omega_{0}^{2}$ is the static polarizability. $T_{33}^{2}$ is

$$
T_{33}^{2}=\frac{e^{-2 \pi x n}}{4 \pi^{2} \rho^{6}}\left[1+2 \pi n x+3(\pi n x)^{2}+2(\pi n x)^{3}+(\pi n x)^{4}\right]
$$

where

$$
x \equiv 2 k_{B} T \rho / \hbar c
$$

and $\rho$ is the distance between the two atoms. In the nonretarded limit $T_{33}^{2} \approx 1 /\left(4 \pi^{2} \rho^{6}\right)$. One can obtain a very similar expression for the Casimir-Polder interaction in free space (cf. Eq. (5) of Ref. [7]). Comparison immediately shows that in the nonretarded limit the free energy of attraction will, regardless of temperature, be reduced to $2 / 3$ of the corresponding free space value. This agrees with the result found at zero temperature by Mahanty and Ninham. They found that the nonretarded interaction in the close proximity of a conducting surface is reduced to $2 / 3$ of the London result in free space, or

$$
F(\rho, T=0 \quad \mathrm{~K})=-\frac{\hbar \omega_{0} \alpha_{0}^{2}}{2 \rho^{6}} .
$$

In other words, the interaction energy is equal to that between a pair of two-dimensional harmonic oscillators.

For large values of $x$ the $n=0$ term in the frequency summation dominates. This occurs at sufficiently high temperatures, or for any finite temperature at sufficiently large separations. The $n=0$ term in the frequency summation becomes reduced in exactly the same way as the nonretarded interaction

$$
F_{n=0}(\rho, T)=-\frac{2 k_{B} T \alpha_{0}^{2}}{\rho^{6}}
$$


Casimir and Polder showed that the interaction energy (at $0 \mathrm{~K}$ ) between two ground-state atoms at separations much larger than $c / \omega_{0}$ vanishes much faster with separation than in the nonretarded limit. In this limit we find that the CasimirPolder interaction on the surface becomes (26/23) of the corresponding result in free space

$$
F(\rho, T=0 \mathrm{~K}) \approx-\frac{13 \hbar c \alpha_{0}^{2}}{2 \pi \rho^{7}} .
$$

The conventional interpretation of the much more rapid decay of the interaction at large separations is related to the loss of interatomic correlation at large separations. However, for any finite temperature, the long-range retarded interaction changes drastically, and it is evident that in practice there is more to it. The interaction will, at large enough separations and finite temperatures, approach a classical analog (where the fluctuations are purely thermal) that does not depend on the velocity of light.

The frequency summation can be performed exactly when retardation is neglected. Using Eq. (14) of Ref. [6] we find that the result is

$$
F(\rho, T)=-\frac{4 \alpha_{0}^{2} k_{B} T}{\rho^{6}}\left[\frac{1}{2}+z \operatorname{coth}(z) / 4+z^{2} \operatorname{csch}^{2}(z) / 4-1 / 2\right],
$$

where $z=\pi / \sqrt{A}$. This is once again exactly $2 / 3$ of the result in free space.

At any temperature the zero-frequency term is cancelled out exactly by contributions from the $n>0$ terms and the leading term is the $T=0 \mathrm{~K}$ interaction energy. In the hightemperature limit a term identical to the $n=0$ term will dominate, and the first correction comes from the $n=1$ term.

The case that we are interested in is when both retardation and finite temperature are included. There will then be a competition between three different length scales: $\rho$ (separation), $c / \omega_{0}$ (related to the atomic resonance) and $c / \xi_{1}$ (related to the thermal energy). We will consider different limiting cases. We first consider the high-temperature limit $\left(\xi_{1}\right.$ $\left.\gg \omega_{0}\right)$. Here we can assume that $A n^{2} \gg 1$ for any finite $n$ and the free energy becomes approximately

$$
\begin{aligned}
F_{n>0} \approx & -\frac{4 e^{4} k_{B} T}{m^{2} \rho^{2} c^{4}}\left[\frac{\operatorname{polylog}\left(4, e^{-2 \pi x}\right)}{(\pi x)^{4}}+\frac{2 \operatorname{polylog}\left(3, e^{-2 \pi x}\right)}{(\pi x)^{3}}\right. \\
& +\frac{3 \operatorname{polylog}\left(2, e^{-2 \pi x}\right)}{(\pi x)^{2}}-\frac{2 \ln \left(1-e^{-2 \pi x}\right)}{\pi x} \\
& \left.+\left(e^{2 \pi x}-1\right)^{-1}\right] \\
& \operatorname{polylog}(n, z)=\sum_{k=1}^{\infty} z^{k} / k^{n} .
\end{aligned}
$$

This expression can be series expanded for both small and large values of $x$. In the limit of large separation, or large $x$, the interaction free energy approaches

$$
\begin{aligned}
F_{n>0} \approx & -\frac{4 e^{4} k_{B} T}{m^{2} \rho^{6} \xi_{1}^{4}}\left[1+2(\pi x)+3(\pi x)^{2}+2(\pi x)^{3}\right. \\
& \left.+(\pi x)^{4}\right] e^{-2 \pi x} \\
\approx & -\frac{4 e^{4} k_{B} T}{m^{2} c^{4} \rho^{2}} e^{-4 \pi k_{B} T \rho / \hbar c} .
\end{aligned}
$$

Thus at large enough separations, the entropy driven $n=0$ term dominates completely in the high-temperature limit. In the limit of small separations and high temperatures we find

$$
F_{n>0} \approx-\frac{2 \pi^{4} e^{4} k_{B} T}{45 m^{2} \rho^{6} \xi_{1}^{4}} .
$$

This term has the same separation dependence as the $n=0$ term but vanishes with temperature as $T^{-3}$. Thus, in this limit as in the free space case, the interaction has one part that has an entropic origin and one part that originates from the internal energy,

$$
E=F-T \partial F / \partial T \approx-\frac{8 \pi^{4} e^{4} k_{B} T}{45 m^{2} \rho^{6} \xi_{1}^{4}} .
$$

When Ninham and Parseigan [41] investigated the interaction free energy between water surfaces, they found that changes in both entropy and enthalpy contributed in the high-temperature limit. This should not be confused with the present result. The dependence on the enthalpy in that case was due to the temperature dependence of the dielectricresponse function. That kind of effect is not considered here.

We next consider thermal corrections to the CasimirPolder result in the low-temperature limit. This will give the lowest-order correction to the zero-temperature interaction energy. We assume that $A \ll 1$, i.e., that the thermal energy is small compared to the atomic resonance energy $\left(\xi_{1} \ll \omega_{0}\right)$, and that $A n^{2} \ll 1$ for all relevant values of $n$. Due to the exponential term in the expression for the free energy only terms of the order $n \sim 1 / 2 \pi x$ contributes significantly. This later approximation is valid when $v \equiv c /\left(\omega_{0} \rho\right) \ll 1$. This simply corresponds to replacing the frequency dependent atomic polarizability with the static polarizability as is usually done to obtain the zero-temperature Casimir-Polder asymptote. We find that

$$
\begin{aligned}
F_{n>0} \approx & \frac{-4 \alpha_{0}^{2} k_{B} T}{\rho^{6}}\left[\frac{1}{e^{2 \pi x}-1}+\frac{2 \pi x e^{2 \pi x}}{\left(e^{2 \pi x}-1\right)^{2}}\right. \\
& +\frac{3 \pi^{2} x^{2} e^{2 \pi x}\left(e^{2 \pi x}+1\right)}{\left(e^{2 \pi x}-1\right)^{3}} \\
& +\frac{2 \pi^{3} x^{3} e^{2 \pi x}\left(1+4 e^{2 \pi x}+e^{4 \pi x}\right)}{\left(e^{2 \pi x}-1\right)^{4}} \\
& \left.+\frac{\pi^{4} x^{4} e^{2 \pi x}\left(1+11 e^{2 \pi x}+11 e^{4 \pi x}+e^{6 \pi x}\right)}{\left(e^{2 \pi x}-1\right)^{5}}\right] .
\end{aligned}
$$


This expression can be expanded for both small and large values of $x$ in the limit of low temperatures and not too large separations (separation large compared to $c / \omega_{0}$ ) but small compared to $\hbar c /\left(k_{B} T\right)$. The free energy then approaches

$$
F(\rho, T) \approx-\frac{13 \hbar c \alpha_{0}^{2}}{2 \pi \rho^{7}}+\frac{16 \alpha_{0}^{2} k_{B}^{4} T^{4} \pi^{3}}{45 \hbar^{3} c^{3} \rho^{6}}+\cdots .
$$

One should note the high order of the first thermal correction $\left(\propto T^{4}\right)$.

At large enough separations the $F_{n>0}$ asymptote becomes

$$
F(\rho, T)_{n>0} \approx-\frac{64 \alpha_{0}^{2} k_{B}^{5} T^{5}}{\rho^{2} \hbar^{4} c^{4}} e^{-4 \pi \rho k_{B} T / \hbar c} .
$$

Of course, in this limit it is better to use the $n=1$ term as the first correction to the $n=0$ contribution, since in this way we avoid any assumptions about the magnitude of atomic resonance frequency. The long-range retarded interaction is for any finite temperature given by the $n=0$ term in the frequency summation.

We finally note that the dispersion contribution to the correlation of atoms in a liquid [42-44] at a liquid-metal interface can be obtained in an entirely analogous manner with results that are expected to be very similar in limiting cases. To conclude this section analogous to the free space case there is a close relationship between temperature and retardation. The interaction between two atoms at a surface is quite similar to the interaction in free space. In the nonretarded limit the interaction is, regardless of temperature, reduced to exactly $2 / 3$ of the interaction in free space. When retardation is included the dominating term in the hightemperature limit will be reduced in the same way. The retarded Casimir-Polder asymptote found at zero temperature is instead enhanced by a factor $26 / 23$. We have finally investigated corrections to these limiting results.

\section{ATOMS IN THE MIDDLE OF A THIN SLIT BETWEEN METALLIC SURFACES}

We next consider the interaction between two atoms in the middle of a very thin slit between two metallic surfaces. Due to strong confinement the interaction will be very different from the previous case. In particular the nonretarded van der Waals interaction decays exponentially and becomes dominated by the retarded Casimir-Polder interaction.

We limit the investigation to slit widths $(L)$ much smaller than both the particle separation and $c / \xi_{n}$ (for all relevant frequencies). Taking $r_{3}=r_{3}^{\prime}=L / 2, r_{2}=r_{2}^{\prime}$, and $\rho=r_{1}-r_{1}^{\prime}$ $>0$, all off-diagonal elements in the Green's function $\mathcal{G}$ vanish. The trace of the Green's functions that we need to evaluate to obtain the free energy of interaction now becomes $\operatorname{Tr}\left[\mathcal{G}\left(\mathbf{R}_{1}, \mathbf{R}_{2}\right) \mathcal{G}\left(\mathbf{R}_{2}, \mathbf{R}_{1}\right)\right]=T_{11}^{2}+T_{22}^{2}+T_{33}^{2}$ where

$$
T_{11}=\frac{1}{L \sqrt{2 \pi}} \sum_{p=1}^{\infty} \sin ^{2}(p \pi / 2) \frac{e^{-\rho \gamma_{p}}}{\sqrt{\rho \gamma_{p}}}\left[\frac{\xi_{n}^{2}}{c^{2}}-\frac{3+4 \gamma_{p} \rho+4 \gamma_{p}^{2} \rho^{2}}{4 \rho^{2}}\right] \text {, }
$$

$$
\begin{gathered}
T_{22}=\frac{1}{L \sqrt{2 \pi}} \sum_{p=1}^{\infty} \sin ^{2}(p \pi / 2) \frac{e^{-\rho \gamma_{p}}}{\sqrt{\rho \gamma_{p}}}\left[\frac{\xi_{n}^{2}}{c^{2}}+\frac{1+2 \gamma_{p} \rho}{2 \rho^{2}}\right], \\
T_{33}=\frac{1}{L \sqrt{2 \pi}} \sum_{p=1}^{\infty}\left(\frac{p \pi}{L}\right)^{2} \cos ^{2}(p \pi / 2) \frac{e^{-\rho \gamma_{p}}}{\sqrt{\rho \gamma_{p}}} \\
+\frac{1}{L \sqrt{2 \pi}} \sum_{p=0}^{\infty} \cos (p \pi) \frac{\xi_{n}^{2}}{c^{2}} \frac{e^{-\rho \gamma_{p}}}{\sqrt{\rho \gamma_{p}}} .
\end{gathered}
$$

Here $\gamma_{p}=\sqrt{\left(\xi_{n} / c\right)^{2}+(p \pi / L)^{2}}$. The $p=0$ term in the last summation is alone responsible for the long-range CasimirPolder-like asymptote found at zero temperature. As will be discussed later the reason for this is that all other terms will have exponentially decaying prefactors. Remembering our initial assumptions, i.e., that the atoms are assumed to be far enough apart in the thin slit, the series consists of terms that go exponentially towards zero with increasing values of $p$. To obtain asymptotic results we, therefore, only retain the $p \leqslant 1$ terms in the summations. We find that $T_{22}$ becomes negligible in comparison to $T_{11}$,

$$
T_{11}^{2}+T_{22}^{2} \approx \frac{\pi^{3} e^{-2 \rho \gamma_{1}}}{2 L^{6} \gamma_{1} \rho} .
$$

Both the nonretarded interaction and the zero-frequency contribution originates from $T_{11}$. The matrix element $T_{33}$ is given by

$$
T_{33} \approx \frac{\xi_{n}^{2}}{c^{2} L \sqrt{2 \pi}}\left[\frac{e^{-\rho \xi_{n} / c}}{2 \sqrt{\rho \xi / c}}-\frac{e^{-\rho \gamma_{1}}}{\sqrt{\rho \gamma_{1}}}\right] .
$$

The Casimir-Polder-like asymptote found at $0 \mathrm{~K}$ comes from the first term of this expression $\left(T_{C P}^{2}\right.$ $\left.\equiv \xi_{n}^{3} e^{-2 \rho \xi_{n} / c} /\left[8 \pi \rho L^{2} c^{3}\right]\right)$. To proceed we series expand $\rho \gamma_{1}$

$$
\rho \gamma_{1}=(\rho \pi / L) \sqrt{1+u^{2}} \approx(\rho \pi / L)\left(1+u^{2} / 2\right),
$$

where we have assumed that $u \equiv 2 k_{B} T L n /(\hbar c) \ll 1$ and that $\rho / L \gg 1$. It is clear that all exponents that contain this term, or similar terms, will decay exponentially, i.e., all terms except that which gives rise to the Casimir-Polder-like asymptote. At zero temperature one finds that the nonretarded interaction decays exponentially,

$$
F(\rho, T=0 \quad \mathrm{~K}) \approx-\frac{2 \pi^{5} \hbar e^{4}}{m^{2} \omega_{0}^{3} L^{6}} \frac{e^{-2 \pi \rho / L}}{2 \pi \rho / L} .
$$

The retarded Casimir-Polder-like asymptote is

$$
F(\rho, T=0 \quad \mathrm{~K}) \approx \frac{-3 \hbar c \alpha_{0}^{2}}{8 L^{2} \rho^{5}} .
$$


For small separations one can no longer neglect the atomic resonances. In the limit when $v\left[\equiv c /\left(\omega_{0} \rho\right)\right]$ goes to infinity, we obtain the following asymptote:

$$
F(\rho, T=0 \quad \mathrm{~K}) \approx \frac{-2 \hbar e^{4}}{\rho L^{2} c^{3} m^{2}} .
$$

One must remember that the interaction in this limit receives important contributions not only from $T_{C P}$. We will return to this in the next section when we discuss a few numerical results. In a bounded region the interaction at small separations decays exponentially, whereas at large enough separations it follows a power law similar to the Casimir-Polder force in free space. Recalling our initial assumption, that the atoms are further apart than the width of the slit, the interaction will be dominated by the Casimir-Polder asymptote for almost every relevant case. More curious still, the interaction in the large separation limit is enhanced by a factor of $3 \pi \rho^{2} /\left(52 L^{2}\right)$ compared to the Casimir-Polder result in free space. Since we assumed that $\rho \gg L$ this is an enormous amplification of the interaction. However, as for the interaction in free space, and on a metal surface, the long-range retarded interaction is fundamentally different for finite temperatures. Replacing the polarizability with its static limit we obtain the Casimir-Polder-like free energy:

$$
F_{C P}(\rho, T) \approx \frac{-2 \pi \alpha_{0}^{2} k_{B} T \xi_{1}^{3}}{\rho L^{2} c^{3}} \sum_{n=0}^{\infty}{ }^{\prime} n^{3} e^{-n 2 \pi x} .
$$

This sum can be evaluated exactly. In the limit of small $x$ it becomes

$$
F_{C P}(\rho, T) \approx-\frac{3 \hbar c \alpha_{0}^{2}}{8 L^{2} \rho^{5}}-\frac{2 \pi^{4} \alpha_{0}^{2} k_{B}^{4} T^{4}}{15 \rho L^{2} \hbar^{3} c^{3}} .
$$

In exactly the same way as for the interaction on a surface the first correction to the Casimir-Polder like asymptote is proportional to $T^{4}$. For the interaction in the midplane between metal surfaces the thermal, or entropy, contributions increase the attraction, similar to in free space. This should be contrasted with what we found for two atoms confined in two dimensions near a surface where the thermal correction decreased the attraction. One should observe that although the overall entropy of a system (including separation independent black-body radiation) must be positive, this is certainly not true for particular interactions.

In the opposite limit $(x \gg 1)$ we find that the interaction decreases exponentially,

$$
F_{C P}(\rho, T) \approx-\frac{16 \pi^{4} \alpha_{0}^{2} k_{B}^{4} T^{4}}{\rho L^{2} \hbar^{3} c^{3}} e^{-4 \pi k_{B} T \rho / \hbar c} .
$$

The Casimir-Polder-like asymptote decays exponentially for any finite temperature at large enough separations. This in contrast to the power law found for the high-temperature limit on a metal surface or in free space. This is the true long-range retarded asymptote for the interaction between two atoms in the middle of a thin slit with metallic walls for most relevant temperatures. However, there will still be a region, at large separations and sufficiently small temperatures, where the interaction follows the zero-temperature Casimir-Polder-like asymptote. At $300 \mathrm{~K} x$ does not exceed unity until separations exceeding $4 \mu \mathrm{m}$. The zerotemperature Casimir-Polder asymptote is often a good approximation at separations less than $1 \mu \mathrm{m}$. However, ultimately at large separations, the interaction decays exponentially.

What about the $n=0$ term in the frequency summation? One might expect this term to give the dominating contribution to the long-range interaction in a similar fashion to that for the interaction in free space and on a surface. In this case it becomes

$$
F_{n=0}(\rho, T)=\frac{-4 \pi^{4} \alpha_{0}^{2} k_{B} T e^{-2 \rho \pi / L}}{L^{5} \rho} .
$$

This term, which does not contain Planck's constant, depends very much on the ratio between particle separation and slit width. In the limit of very narrow slit width we can neglect this term compared to the Casimir-Polder asymptote for any relevant temperatures. The terms that we have neglected can similarly be shown to be small in the limits that we have considered.

We can again see the important relationship between distance and temperature for the long-range interaction between atoms. However, the long-range asymptote for finite temperatures can no longer be found by simply taking the $n$ $=0$ term in the frequency summation. Comparing the exponents of Eqs. (43) and (44), it is clear that the $n=0$ asymptote dominates once $\hbar c /\left(2 k_{B} L\right) \ll T$. At a temperature of $1000 \mathrm{~K}$ this means that the slit width must be greater than approximately $1 \mu \mathrm{m}$. For the examples that we consider here, the long-range Casimir-Polder asymptote is the dominating by far. In any case the true long-range interaction decays away exponentially at any finite temperature. The inclusion of one extra length scale, i.e., the slit width, is of course the origin of the new features found in this final example. In the opposite limit when $\rho / L \ll 1$ other approximations must be used to obtain correct analytical expressions. The summations in the Green's functions can be replaced with integrations and the $n=0$ term becomes

$$
F_{n=0}(\rho, T)=-\frac{3 k_{B} T \alpha_{0}^{2}}{\rho^{6}}\left[1-\frac{\rho^{3}}{3 L^{3}}-\frac{\rho^{5}}{2 L^{5}}+\cdots\right] .
$$

With increasing slit width the interaction in the midplane between two metal surfaces approaches the free space result as it should.

\section{NUMERICAL RESULTS}

We have numerically investigated the interaction at 1000 $\mathrm{K}$ between two hydrogen atoms on a metal surface, and in the midplane of a narrow cavity with metallic walls. The high temperature is relevant for catalysis of atoms on surfaces, narrow slits, and in pores. The parameters used to model the polarizability of a hydrogen atom were taken from 


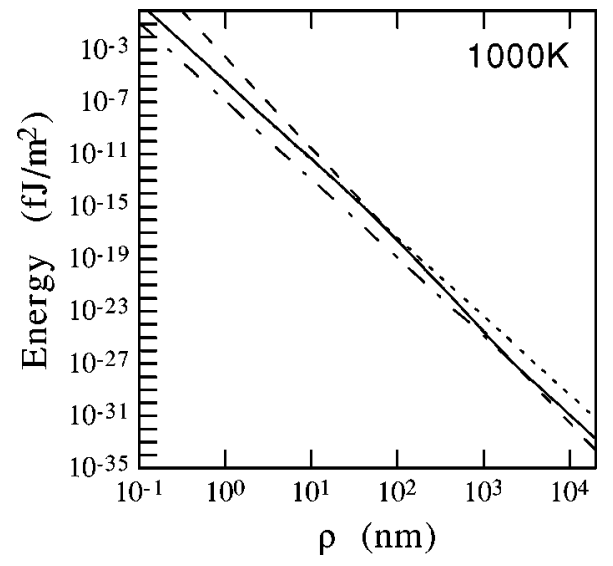

FIG. 1. The interaction free energy at $1000 \mathrm{~K}$ between two atoms on a metal surface (solid line) is compared with different asymptotes: the $0 \mathrm{~K}$ Casimir-Polder asymptote (dashed), the nonretarded van der Waals asymptote (dotted), and the $n=0$ asymptote (dashed dotted).

Rauber et al. [45]. We used a static polarizability of 4.50 atomic units $\left(1\right.$ a.u. $=1.482 \times 10^{-29} \mathrm{~m}^{3} /$ atom $)$ and a characteristic absorption frequency of $11.65 \mathrm{eV}$.

In Fig. 1 we compare the interaction free energy between two hydrogen atoms on a metal surface with different asymptotes. We have previously shown that there are smooth transitions between different separation regions for the interaction between an atom and two metallic sheets [46]. We can, in an analogous manner, get a fairly good approximation of the interaction free energy by simply using the nonretarded van der Waals asymptote for small separations, the Casimir-Polder asymptote at larger separations, and finally at even larger separations the high-temperature asymptote (the $n=0$ term). For $T=1000 \mathrm{~K}, \hbar c /\left(k_{B} T\right)=2.3 \mu \mathrm{m}$ so thermal effects will begin to influence the interaction unless the separation is small compared to $1 \mu \mathrm{m}$. For future reference we note that the interaction increases with temperature being almost entirely entropic at large enough separations.

As we have already stated the interaction in a narrow slit is very different from the interaction in free space, or on a surface. In Fig. 2 the interaction free energy in a very narrow cavity is compared with different asymptotes. One should note that $F_{n=0}$ is totally negligible even at this high temperature. In order for this part of the interaction to dominate one has to increase the temperature at least one order of magnitude. At very small separations one can no longer assume that the particle separation is much smaller than the slit width and one can no longer expect the interaction to follow our nonretarded asymptote. At slightly larger, but still small, separations the interaction becomes dominated by the nonretarded asymptote that originates from $T_{11}$, but there will also be minor contributions from the other terms. At intermediate separations, where the high-frequency spectrum still dominates, the interaction follows the small separation CasimirPolder-like asymptote, which depends on the atomic resonance. The actual interaction found numerically at these separations is smaller than the asymptote given in the previous section. The reason is that many terms of alternating sign in the second sum in $T_{33}$ contributes. At even larger separa-

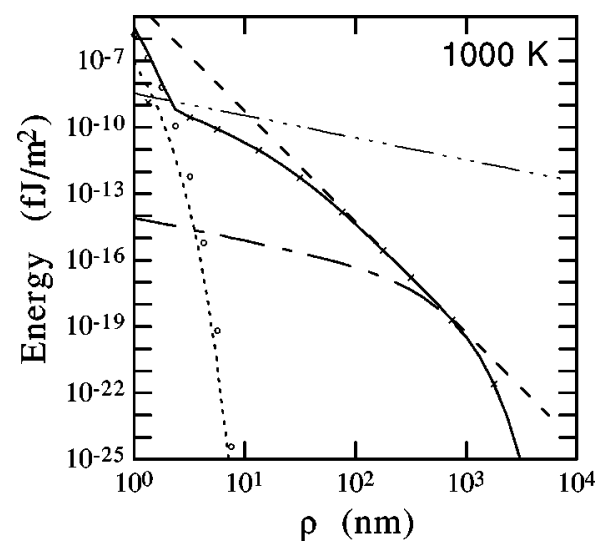

FIG. 2. The interaction free energy (solid line) at $1000 \mathrm{~K}$ between two atoms in the midplane of a metallic cavity $(\mathrm{L}=1 \mathrm{~nm})$ is compared with different asymptotes: the $0 \mathrm{~K}$ Casimir-Polder asymptote (dashed), the high-temperature Casimir-Polder asymptote (dashed-dotted), the nonretarded van der Waals asymptote (circles), the $n=0$ asymptote (dotted), the energy contribution that comes from the second sum in $T_{33}$ (crosses), and finally the small separation Casimir-Polder asymptote (dashed-triple dotted).

tions, where the relevant frequencies become much smaller than the atomic resonance frequency, the interaction follows the long-range $0 \mathrm{~K}$ Casimir-Polder-like asymptote. At still larger separations the interaction follows the hightemperature Casimir-Polder asymptote. We note that the interaction is totally dominated by the contributions that come from the second sum in $T_{33}$ at separations exceeding $10 \mathrm{~nm}$.

In Fig. 3 we explore the ratio between the interaction energy to the interaction free energy between two atoms in free space, on a surface, and finally in a narrow slit. At large separations the interaction between two atoms in free space, and on a surface, has a purely entropic origin. For these two cases the ratio goes, as expected, to zero at large separations. One should note that it may exceed unity in a small separation range for the interaction on a surface. As might be expected the more interesting case is the interaction in a narrow

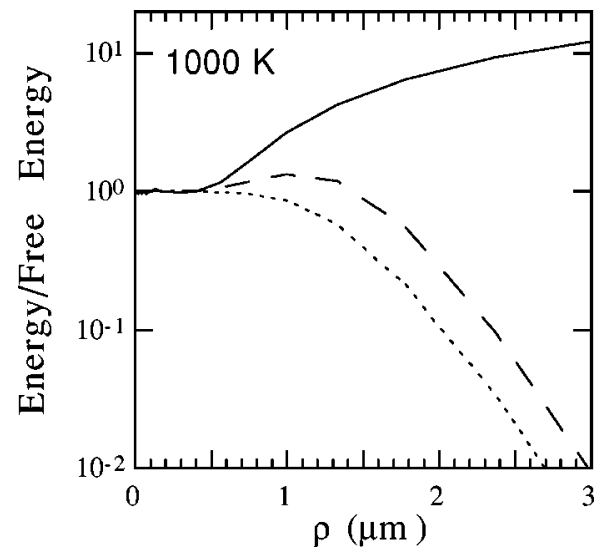

FIG. 3. Ratio between the energy and the free energy for two atoms in free space (dotted), on a metal surface (dashed), and in a 1-nm slit with metal walls (solid). The free energy was evaluated at $1000 \mathrm{~K}$, and the energy was obtained from a discretization of Eq. (28) with $F$ evaluated at 950,1000 , and $1050 \mathrm{~K}$. 


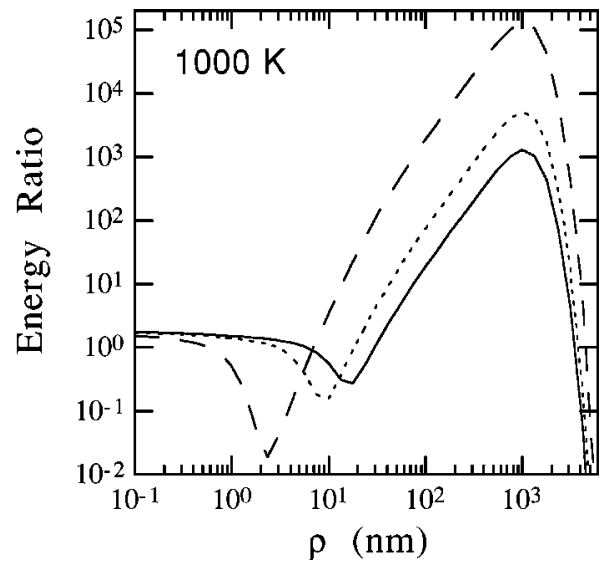

FIG. 4. The ratio between the interaction free energy at $1000 \mathrm{~K}$ between two atoms in a slit to the corresponding energy in free space. Three different slit widths were considered: $1 \mathrm{~nm}$ (dashed), 5 $\mathrm{nm}$ (dotted), and $10 \mathrm{~nm}$ (solid).

cavity. Using the relation between energy, entropy, and free energy, and the expression for the high-temperature CasimirPolder asymptote, we obtain the following relations:

$$
\begin{aligned}
& E / F \approx-3+4 \pi k_{B} T \rho /(\hbar c), \\
& -T S / F \approx 4-4 \pi k_{B} T \rho /(\hbar c) .
\end{aligned}
$$

This is totally different from the asymptotic interaction on a surface $\left(F=-T S=-2 k_{B} T \alpha_{0} / \rho^{6}\right)$ where the internal energy goes to zero. One may compare with the hightemperature limit of the interaction between an atom and metallic sheets [46]. There was a large cancellation between thermal corrections that originated from different modes. The part that originated from transverse magnetic modes increased with temperature in contrast to the part that came from transverse electric modes, which decreased with temperature. Due to this cancellation thermal effects on the total interaction occured at much larger separations than for the separate parts. It is noteworthy that only the transverse magnetic contributions had a linear temperature dependence at high $T$, i.e., purely entropic. The separation dependence of the different contributions were thus different also in that case.

Finally, Fig. 4 presents the ratio between the free energy of interaction in a slit to the corresponding interaction in free space. In the limit of small separations the interaction in the slit is dominated by nonretarded contributions that come mainly from $T_{11}$. The modes that dominates this part of the interaction are exponentially damped in the slit already at very small separations. The result is the lowering of the interaction compared to in free space that can be seen at separations around 1-10 nm. At larger separations the retarded Casimir-Polder part of the interaction dominates. For slit widths of 1,5 , and $10 \mathrm{~nm}$ the enhancement of the interaction in this limit due to confinement may be as large as $10^{3}-10^{5}$ times. This effect decreases with increasing slit width but it will still influence the interaction in substantially larger slits. At separations exceeding $0.1 \mu \mathrm{m}$ thermal effects become important. There is a sharp drop in the energy ratio when the interaction follows Eq. (43). In this limit all relevant electromagnetic modes propagates as evanescent waves through the narrow channel.

\section{CONCLUSIONS}

We have investigated the interaction between two atoms at a metal surface, and in a thin slit with metallic, perfectly conducting, walls.

We have found Casimir-Polder-like long-range asymptotes at zero temperature. However, in many situations thermal effects are important for any nonzero temperature at large enough separations. While the zero temperature results are not useless, they are of limited validity and it is important to know limitations of such approximations.

The interaction between two atoms on a conducting surface goes asymptotically towards the classical interaction between a pair of two-dimensional harmonic oscillators. This interaction is not due to the $n=0$, or classical term, but in the same way as for the free space case [7] it is due to thermal population of the electromagnetic field modes.

To see this one can simply consider the nonretarded limit: here, regardless of temperature, the $n=0$ term is cancelled out exactly by one term that comes from the sum of $n>0$ terms. At high temperatures when the field modes become thermally excited, the $n>0$ terms produce a term that is identical to the $n=0$ term.

This demonstrates once again the correspondence principle that a quantum system goes over to its classical analog when the relevant photon modes are fully excited. It is remarkable that this is not only true for high (compared to the resonance frequency) temperatures, but it also often occurs at large separations.

The change in power law at large separations has usually been interpreted as being simply due to the finite velocity of light. However, the long-range interaction at finite temperatures between two atoms on a surface (or in free space as in Ref. [7]) is independent of the velocity of light. This shows that there is more to it than a simple loss of intercorrelation due to the finite velocity of light. As pointed out by Wennerström et al. [7] the quantum nature of light is important to the softening of the interaction potential.

In the middle of a narrow slit with conducting walls the long-range interaction between two atoms decreases exponentially at any finite temperature. This is because the modes that contribute to the classical term are suppressed in a cavity. If the slit is too narrow, light of the appropriate frequencies can only propagate as exponentially decaying evanescent waves. These modes, therefore, fail to dominate the free energy through entropic contributions in the presence of atoms. This is similar to the case of dispersion forces in the presence of electrolytes [13] where Debye screening causes an exponential decay to the interaction. The long-range interaction need no longer be the same as the $n=0$ term. In fact, it is only when high temperature is large compared to $\hbar c /\left(2 k_{B} L\right)$ that the long-range interaction has a pure entropic origin in this case. For most relevant temperatures the long-range interaction follows Eq. (43). Changes in both entropy and internal energy contribute to this asymptote. To 
conclude, in extremely confined regions one can no longer expect the long-range Casimir-Polder interaction to always go over in a simple way to the corresponding "classical" limit.

It is straightforward to describe the interaction between two atoms at arbitrary positions in a narrow slit. The Green's functions will in general have nonzero off-diagonal matrix elements. The contributions that originate from these will decay in much the same way as the contributions that come from $T_{11}$ and $T_{22}$. The second sum in $T_{33}$, which give rise to the Casimir-Polder-like asymptote, become modulated by a term $\cos \left[n \pi\left(z+z^{\prime}\right) / L\right]$. Since it is the $n=0$ term that give rise to the long-range Casimir-Polder asymptote this interaction is actually independent of $z$ and $z^{\prime}$ in narrow cavities and given by Eq. (43).

\section{ACKNOWLEDGMENTS}

M.B. would like to acknowledge financial support from the Australian Research Council and from STINT, the Swedish Foundation for International Cooperation in Research and Higher Education.
[1] H.B.G. Casimir, Proc. K. Ned. Akad. Wet. 51, 793 (1948).

[2] H.B.G. Casimir and D. Polder, Phys. Rev. 73, 360 (1948).

[3] B.W. Ninham and V. Yaminsky, Langmuir 13, 2097 (1997).

[4] M. Boström, D.R.M. Williams, and B.W. Ninham, Langmuir 17, 4475 (2001).

[5] M. Boström, D.R.M. Williams, and B.W. Ninham, Phys. Rev. Lett. (to be published).

[6] B.W. Ninham and J. Daicic, Phys. Rev. A 57, 1870 (1998).

[7] H. Wennerström, J. Daicic, and B.W. Ninham, Phys. Rev. A 60, 2581 (1999).

[8] I. Lundström, P. Norberg, and L.-G. Petersson, J. Appl. Phys. 76, 142 (1994).

[9] P. Norberg, U. Ackelid, I. Lundström, and L.-G. Petersson, J. Appl. Phys. 81, 2094 (1997).

[10] Y. Nishi, T. Suzuki, and K. Kaneko, J. Phys. Chem. B 101, 1938 (1997).

[11] Z. Blum, S.T. Hyde, and B.W. Ninham, J. Phys. Chem. 97, 661 (1993).

[12] J. Mahanty and B.W. Ninham, J. Phys. A 6, 1140 (1973); 5, 1447 (1972).

[13] J. Mahanty and B.W. Ninham, Dispersion Forces (Academic, London, 1976), and references therein.

[14] J.-M. Vigoureux, P. Grossel, D.V. Labeke, and C. Girard, Phys. Rev. A 35, 1493 (1987).

[15] H. Nha and W. Jhe, Phys. Rev. A 54, 3505 (1996).

[16] M. Cho and R.J. Silbey, J. Chem. Phys. 104, 8730 (1996).

[17] M. Cho, J. Chem. Phys. 110, 4998 (1999).

[18] J. Mahanty and B.W. Ninham, Phys. Lett. 43A, 495 (1973).

[19] M. Boström, J.J. Longdell, D.J. Mitchell, and B.W. Ninham, Phys. Rev. Lett. (to be published).

[20] P.W. Milonni, The Quantum Vacuum (Academic, New York, 1993).

[21] S.K. Lamoreaux, Phys. Rev. Lett. 78, 5 (1997); 81, 5475 (1998).

[22] U. Mohideen and A. Roy, Phys. Rev. Lett. 81, 4549 (1998).
[23] A. Roy, C.-Y. Lin, and U. Mohideen, Phys. Rev. D 60, 111101 (1999).

[24] A. Shi, D. Raskin, and P. Kusch, Phys. Rev. A 9, 652 (1974).

[25] A. Shi, Phys. Rev. A 9, 1507 (1974).

[26] A. Shi and V.A. Parseigan, Phys. Rev. A 12, 835 (1975).

[27] A. Anderson, S. Haroche, E.A. Hinds, W. Jhe, and D. Meshede, Phys. Rev. A 37, 3594 (1988).

[28] V. Sandoghdar, C.I. Sukenik, E.A. Hinds, and S. Haroche, Phys. Rev. Lett. 68, 3432 (1992).

[29] C.I. Sukenik, M.G. Boshier, D. Cho, V. Sandoghdar, and E.A. Hinds, Phys. Rev. Lett. 70, 560 (1993).

[30] M. Bordag, B. Geyer, G.L. Klimchitskaya, and V.M. Mostepanenko, Phys. Rev. D 58, 075003 (1998).

[31] G.H. Goedecke and R.C. Wood, Phys. Rev. A 60, 2577 (1999).

[32] T. Boyer, Phys. Rev. A 11, 1650 (1972).

[33] M.J. Mehl and W.L. Schaich, Surf. Sci. 99, 553 (1980).

[34] B. Davies and B.W. Ninham, J. Chem. Phys. 56, 5797 (1972).

[35] W.L. Schaich, Phys. Rev. B 31, 3409 (1985).

[36] E.M. Lifshitz, Zh. Éksp. Teor. Fiz. 29, 94 (1955) [Sov. Phys. JETP 2, 73 (1956)].

[37] B.W. Ninham, V.A. Parsegian, and G.H. Weiss, J. Stat. Phys. 2, 323 (1970).

[38] J. Mehra, Physica 37, 145 (1967).

[39] L.S. Brown and G.J. Maclay, Phys. Rev. 184, 1272 (1969).

[40] M. Boström and Bo E. Sernelius, Microelectron. Eng. 51-52, 287 (2000).

[41] V.A. Parseigan and B.W. Ninham, Biophys. J. 10, 664 (1970).

[42] L.D. Landau and E.M. Lifshitz, Statistical Physics Part 2 (Butterworth-Heinemann, Oxford, 1998).

[43] M.P. Kemoklidze and L.P. Pitaevskii, Zh. Éksp. Teor. Fiz. 59, 2187 (1970) [Sov. Phys. JETP 32, 1183 (1971)].

[44] Yu.S. Barash and O.I. Notych, Zh. Éksp. Teor. Fiz. 98, 542 (1990) [Sov. Phys. JETP 71, 301 (1990)].

[45] S. Rauber, J.R. Klein, M.W. Cole, and L.W. Bruch, Surf. Sci. 123, 173 (1982).

[46] M. Boström and Bo E. Sernelius, Phys. Rev. A 61, 052703 (2000). 